\title{
A Brief Introduction
}

Abstract In this chapter we introduce the themes of the book and present our overall approach to clinical ethics for health professionals. Of special note is our assumption that the social sciences-especially history, sociology, and anthropology/psychology—can do much to enrich how health professionals think about clinical ethics. We also provide an overview of the book as a whole.

Keywords Health care - Clinical ethics - Bioethics - Ethical principles Health professionals - Patients - Social sciences - Professional education

The audience for this book is anyone who has experienced a discrepancy between their own individual thinking about ethics-whether in medicine, nursing, social work, psychology, or other fields-and what they encounter in the academically oriented, comparatively theoretical discussions of ethics as presented in grand rounds, at conferences, and in professional and academic journals. What these clinicians know is that this difference makes a difference: their ways of thinking and acting are grounded not in academic abstractions but in their own selves, their general life experiences, years of clinical encounters with patients, and myriad discussions with friends and colleagues, both junior and senior. What they also know is that when they need advice concerning ethical problems in their work, the best source of support and feedback is likely

(C) The Author(s) 2018

S. Scher and K. Kozlowska, Rethinking Health Care Ethics, https://doi.org/10.1007/978-981-13-0830-7_1 
to be their own colleagues, who understand how those problems are embedded in, and inseparable from, the clinical milieu.

That, in a nutshell, is what this book is about. The starting point is that health care trainees and clinicians carry around with them, as part of their very selves, the emotional and intellectual resources required for them to act and think ethically-or, in broader terms, humanely and reflectively-in their encounters with patients. Any effort to develop clinically relevant ethics that fails to build upon these preexisting personal resources will inescapably fall short of achieving its intended result, however good or admirable.

\section{The Dominance of Bioethics}

In this context, health professionals have not been well served, or at least not well enough served, by the academic community of bioethicists-the philosophers, theologians, lawyers, and social scientists of various sorts who have come to dominate, worldwide, the diverse fields of health care ethics over the last half century. The theoretical, philosophically oriented approaches of bioethicists generally leave clinicians feeling somewhat at a loss, not knowing exactly how to proceed. Especially noteworthy in this regard is the principle-based approach commonly known as principlism, in which autonomy, beneficence, nonmaleficence, and justice, along with rules such as confidentiality, privacy, and truthfulness, are deemed sufficient to generate and understand all of health care ethics. The models of ethical discourse presented by bioethicists, however, regularly fall short of connecting with the clinical milieu and with clinicians' own welldeveloped, intuitively engaging modes of ethical thinking. And though these latter modes of thinking are the ones with which clinicians are most comfortable, bioethicists have seen these concrete, humanly grounded ways of thinking as too informal and unsystematic to merit serious attention.

Understood in terms of the classic triad of thought, emotion, and action, bioethicists have focused almost exclusively on thought-ethical thinking per se-and given inadequate inattention to emotion and action. What has been lost in the process are the concrete human dimensions of caregiver-patient interactions and, more generally, the connection between ethical discourse and the full dimensions of the self. Especially in a domain of activity, such as health care, that is so rich in human interactions, it is 
only by respecting and building upon the interconnectedness of thought, emotion, and action (Bruner 1986) that one can expect any success in teaching clinicians or otherwise advancing clinical ethics.

One final but crucially important point. Feelings — understood here as the subjective experience of emotion (Damasio 1994) — play a centrally important role in the process of ethical discovery and, indeed, in ethical discourse generally. ${ }^{l}$ Philosophers, because of their probing attention to ethical theory, may learn to access their moral thinking with ease (though as the first author [SS] can well attest, only after years of effort and training), the easiest mode of access for everyone else is often through their gut feelings about right and wrong, about the fittingness or unfittingness of a current or proposed action, or about something being not quite what it should be. These feelings, a deep expression of the self, are a sign that further thought may be required, that something needs to be sorted out. And without these signs, many of us would have no idea where to begin moral exploration.

It is the above elements - the thoughts, emotions (and feelings), and actions of the self, plus a person's professional and personal experiences and goals - that serve as our point of departure. By taking these elements as the foundation for rethinking health care ethics, we hope to develop an approach that embraces and builds upon the particularity of each individual clinician.

\section{The Lens of Social Science}

Another way of understanding our approach is through the lens of social science. As a relatively new and influential social phenomenon, bioethics has drawn the attention of leading social scientists. A common theme raised by social scientists is the narrow intellectual perspective of bioethics, its overly rationalistic modes of analysis, and its disconnection from flesh and blood human beings. ${ }^{2}$ The sociologist Renée Fox (1999, p. 9) notes that bioethics'

coolly rational mode of analysis focused on autonomy-of-self bends [bioethics] away from detailed attention to the empirical contexts in which ethically relevant events occur, from how they are experienced, and from serious consideration of the play of both rational and nonrational social and cultural factors in moral life. 
She adds that bioethics tends not only "to minimize the role of social and cultural factors" but to "regard them as epiphenomena." In another essay, Fox, along with her coauthor Judith Swazey, presents an excellent summary of criticisms of bioethics-some from outside, some from within bioethics itself - many of which focus on the limits of principlism and its inattention to the broader human dimensions of health care (Fox and Swazey 2005).

The same frustration with the narrow frameworks of bioethicists is reflected in the observation by the historians Gary Belkin and Allan Brandt $(2001$, p. 8$)$ that "history can shift our attention to how experiences and practice are established in culture rather than focus on abstracted terms, concepts, and formulations." Indeed, bioethical formulations need to resonate with experience and to capture "how attitudes toward suffering, expectations about medicine, customs of establishing desert and entitlement, get formed, cohere, and change." In a separate essay Belkin (2004, p. 378) urges that bioethics be supplanted by a medical humanism that is

less concerned with generating rules of conduct than with deepening and enriching the self-understanding and perspective brought to bear when people confront choices and each other. And a humanistic ongoing engagement and routine self-reflection can make medicine more deeply ethical than can duels over methodologies of ethics per se. Bioethics has narrowed how reflection in medicine about medicine takes place and has inhibited rather than rescued a medical humanism by an overrated focus on restrictive reduction to "the ethical."

Belkin and Brandt, like Fox, see the need to move past the goals and standards of present bioethics to a broader framework that incorporates cultural, historical, psychological, and social perspectives and that, as Leon Kass (1990, p. 8) argued so lucidly, is strongly oriented not to the analysis of "extreme examples" but to the "morality of ordinary practice."

The anthropologist Arthur Kleinman (1999, p. 72) has focused on the gap between the particular and the universal, between "moral experience," which is local and rich, and the goals and methods of bioethics, with its quest for "objective standards" and its "models of moral reasoning championing the reflection and rational choice of autonomous individuals." Bioethics, in Kleinman's view, "risk[s] irrelevance"; it "simply 
does not account for social processes of moral life." What any viable approach to ethics in health care does need to take into account is the moral experience of individuals, which

is about the local processes (collective, interpersonal, subjective) that realize (enact) values in ordinary living. These processes cross the boundary of the body-self, connecting affect and cognition with cultural meanings, moral norms and collective identity with sense of self. Thus moral experience and personal experience are interfused, value with emotion. (pp. 71-72)

Though Kleinman elaborates an anthropological/ethnographic approach to bridging moral experience and bioethics into some form of workable harmony, the approach we take into this book is broader and more eclectic. Like Kleinman, we see individual moral experience as fundamental. It is crucial in understanding clinical ethics generally-that is, how health professionals understand and address the ethical dimensions of their work. And it is also crucial to the process of professional training in health care-that is, how we help particular individuals make the transition from preprofessional aspirants to professional trainees in health care to mature professionals. In that context, and building upon Kleinman's notion of individual moral experience, the present volume represents our own effort to develop an approach to health care ethics that draws upon the insights of sociology, history, and anthropology, and that bridges the gap between local, concrete experience and the quest for universality and objectivity. ${ }^{3}$

We have been focusing in this section on perspectives from the social sciences, but bioethics has also met with strong criticisms from within. Two of the most articulate such critics have been Daniel Callahan, himself the founder of the Hastings Center (see Chapter 3), and Larry Churchill. ${ }^{4}$ Callahan (2003) is a self-described communitarian, and Churchill's graduate training was in religious studies rather than philosophy; because of these orientations, both are likely more attuned to the human dimensions of health care. By the same token, their critiques have much in common with those of social scientists. Callahan (2003, p. 288) is especially critical of the "all too common" mistake by philosophers that

good ethics comes down to good arguments. It is as if an anatomist thought that human nature could best be understood by stripping all the flesh off a body to uncover the hidden bones. . . Rationality at the least needs the help of the imagination. At the clinical level this means, 
for instance, an ability to enter into the needs, pain, and suffering of others, to grasp their situation and respond appropriately to it. At the policy level it means understanding-for example, how a proposed health care reform might not only improve health or access to health care, but how it could play out in the larger political and social scene.

Our own view, and we're sure that Callahan would agree, is that the enrichment of ethical discourse with materials from the social sciences is a potentially powerful way to facilitate, on both the clinical and policy levels, the "imagination" to which he refers. Much of what we say in our chapters on teaching ethics (Chapters 8-10) is based on this insight, coupled with the idea that teaching needs to engage the whole personthought, emotion, and action—and not just the intellect.

\section{LIMITATIONS}

The field of health care ethics is, of course, vast, and we obviously will be discussing only part of it. What we will not be discussing, except in passing, are questions of public policy, research ethics, legal regulation and professional accountability (including licensure, disciplinary frameworks, and negligence/malpractice), and patient safety, the organized effort to reduce preventable injuries within health care. ${ }^{5}$ With regard to this last topic, the safety of patients is obviously of paramount concern in all fields of health care, and the World Health Organization's efforts to promote patient safety have, on a global scale, raised awareness of, and helped to reduce, preventable injuries. What our book can contribute to patient safety is to help health professionals to recognize the legitimacy of their own ethical thinking, to bring that thinking to bear upon their own work, work environment, and culture, to communicate and cooperate more effectively, and to look critically at-and when necessary, act to improve upon-existing practices.

\section{Chapter Summary}

Chapter 2. The Limitations of Bioethics: A Personal History. The next chapter looks at the origins of this book in the work of the first author [SS] as a researcher, teacher, and consultant in the 1980s, when he taught ethics, at the bedside, in hospitals affiliated with Harvard Medical School. 
Chapter 3. The Rise of Bioethics: A Historical Overview. In this chapter we discuss the rise of bioethics beginning in the late 1960s with the founding of the Hastings Center, followed shortly thereafter by the Kennedy Institute of Ethics at Georgetown University. After looking at its early U.S. history, we turn to the mixed response to the bioethics movement in Europe and the change in direction there following the discovery of Fritz Jahr's work from the first half of the twentieth century. Finally, we look at how the 2005 Universal Declaration on Bioethics and Human Rights fits into this history.

Chapter 4. Theory and Practice: From the Top Down. We turn here to the development of substantive doctrine in bioethics, beginning with the California Supreme Court's 1957 Salgo decision introducing the legal doctrine of informed consent, and moving up to and through the formulation, in the late 1970s, of four central ethical principles for understanding health care ethics. We note some discontinuities between bioethics, so conceived, and the clinical thinking and practice of health professionals, and we raise some questions concerning ethical "dilemmas," closure, and ethics expertise.

Chapter 5. The Elusiveness of Closure. When confronting any difficult ethical situation, reaching closure-a single, determinate decision-is typically difficult. Some bioethicists have proposed that health professionals use multistep processes as a means of ensuring thorough consideration of the relevant issues and reaching a conclusion. To evaluate such proposals, we look at a multistep process that does work: the judicial appeals process as exemplified by U.S. federal courts (which we use as a convenient model). We suggest that various institutional, educational, social, and substantive/intellectual factors are at work in enabling appeals courts to reach decently informed, well-reasoned decisions, and that these factors are not shared by the suggested multistep processes for ethical decision making. We conclude that health professionals need to look elsewhere for a model for making ethical decisions.

Chapter 6. Ethics and the Self. Going back to basics, we see the development of ethical thought, emotion, and action as an inescapable and fundamental part of becoming a person, and as the essential background against which we need to think about clinical ethics for health care trainees and health professionals. We distinguish between formal and informal ethical discourse, noting that bioethicists engage in the former, whereas health professionals engage in the latter. We also introduce the notion of fast and slow thinking as a way of understanding informal 
ethical thinking and what we describe as implicit ethical frameworks, which could be understood as ethics without the language of ethics.

Chapter 7. The Self in an Interpretive Community. We present two vignettes - each of which invokes implicit ethical frameworksas a means of introducing the notion of interpretive communities. This notion, first elaborated by the literary theorist Stanley Fish in his 1980 book Is There a Text in This Class?, enables us to understand how clinical ethics can be understood as embedded - invisibly but powerfully-within health care institutions and practices. As a consequence, health professionals typically discuss clinical ethics not in terms of principles or other express ethical standards, but in terms of goals, values, concerns, complications, benefits, risks, problems, uncertainties, and other factors that potentially affect the care of patients.

Chapter 8. A Framework for Teaching Clinical Health Care Ethics. In this first of three chapters on teaching, we frame the general question as how to extend trainees' existing ethical frameworks to their new professional fields (e.g., medicine, nursing, social work). We assume that trainees, when they enter professional school, already have welldeveloped ethical frameworks of their own. The challenge is to integrate those existing frameworks with trainees' growing knowledge of their own fields - that is, with the process of becoming mature members of their own particular interpretive communities. In this context we discuss what we call touchstones for learning (i.e., questions that help one gain access to one's own ethical thinking), the need to create space for reflection and discussion, and the use and potential abuse of senior clinicians' power and authority to maintain the status quo and discourage professional growth.

Chapter 9. Teaching Clinical Ethics in the Formal Curriculum. In this second of three chapters on teaching, we discuss the design of formal courses for teaching clinical ethics, giving particular attention to core lecture courses, discussion classes, and observational and participatory activities of various sorts. As with the other chapters on teaching, the focus is on the self, informal ethical discourse, and interpretive communities. For our examples, we focus on some of the central substantive issues relevant to the education of health trainees and professionals: mental health, cultural competence (extended), patient communication, informed consent, and shared decision making. We also discuss the use of counterstories as a means of exploring the positions and perceptions of others. 
Chapter 10. Teaching Ethics in a Clinical Setting. In this last of three chapters on teaching, we address the challenges of teaching ethics during the actual clinical portion of professional education. The opportunities for teaching are diverse and even extensive, at least if the institutions make a place for such opportunities by setting aside (and protecting) the time needed for such activities. We discuss the possibility for regularly scheduled case conferences of various kinds, ad hoc case conferences, formal clinical modules, and mentoring. We also briefly discuss the challenges that trainees face in maintaining the self in the face of unrelenting work and the professional and career pressures presented by their interactions with senior clinicians.

Chapter 11. Empowering Clinicians. Weaving together the themes discussed in earlier chapters, this concluding chapter presents a straightforward, relatively informal summary of what we hope clinicians will take away from reading this book. In the process, we attempt to anticipate and clarify some potential questions about what we have tried to communicate. The chapter title, in a way, says it all. Our primary goal is to empower clinicians to respect their own moral thinking and to use it in addressing the problems that they confront in clinical health care.

\section{Notes}

1. Academic writers have spilled large amounts of ink over the proper use of moral versus ethical. For better or worse, moral is more common in philosophical circles, whereas ethics is overwhelmingly more common in health care, presumably because of the recurrent attention paid to codes of professional ethics. For simplicity of expression, we will be using ethical rather moral except when doing so produces obviously odd results or some form of ambiguity. For example, a moral philosopher is a philosopher who focuses on problems in a particular field of philosophy, alternatively known as either moral philosophy or ethics. An ethical philosopher would be a philosopher who acts ethically, independent of the field of his or her specialization.

2. We only scratch the surface here of the criticisms of bioethics from the perspective of the social sciences. See, for example, Leigh Turner's wide-ranging article, "Anthropological and Sociological Critiques of Bioethics" (2009).

3 . For a recent, engaging effort to bridge this gap and move past current criticisms of bioethics, specifically in relation to medicine, see Jing-Bao Nie, Medical Ethics in China: A Transcultural Interpretation (2011).

4. See, for example, Churchill's "Are We Professionals? A Critical Look at the Social Role of Bioethicists" (1999). 
5. The landmark event for the WHO's patient-safety efforts was the 2002 resolution of the World Health Assembly (WHA55.18), in which WHO member states were asked to "pay the closest possible attention to the problem of patient safety and to establish and strengthen science-based systems necessary for improving patients' safety and quality of care." The World Alliance for Patient Safety was launched in 2004; the report Conceptual Framework for the International Classification of Patient Safety was published in 2009; and the two editions of the Patient Safety Curriculum Guide were published in 2009 (specifically for medical schools) and 2011 (for all health professions). For a helpful overview, see "Educating Future Leaders in Patient Safety" (Leotsakos et al. 2014).

\section{REFERENCES}

Belkin, G. S. (2004). Moving beyond bioethics: History and the search for medical humanism. Perspectives in Biology and Medicine, 47(3), 372-385.

Belkin, G. S., \& Brandt, A. M. (2001). Bioethics: Using its historical and social context. International Anesthesiology Clinics, 39(3), 1-11.

Bruner, J. S. (1986). Actual minds, possible worlds. Cambridge, MA: Harvard University Press.

Callahan, D. (2003). Principlism and communitarianism. Journal of Medical Ethics, 29(5), 287-291.

Churchill, L. R. (1999). Are we professionals? A critical look at the social role of bioethicists. Daedalus, 128(4), 253-274.

Damasio, A. R. (1994). Descartes' error: Emotion, reason, and the human brain. New York: G.P. Putnam.

Fox, R. C. (1999). Is medical education asking too much of bioethics? Daedalus, $128(4), 1-25$.

Fox, R. C., \& Swazey, J. P. (2005). Examining American bioethics: Its problems and prospects. Cambridge Quarterly of Healthcare Ethics, 14(4), 361-373.

Kass, L. R. (1990). Practicing ethics: Where's the action? Hastings Center Report, 20(1), 5-12.

Kleinman, A. (1999). Moral experience and ethical reflection: Can ethnography reconcile them? A quandary for "the new bioethics." Daedalus, 128(4), 69-97.

Leotsakos, A., Ardolino, A., Cheung, R., Zheng, H., Barraclough, B., \& Walton, M. (2014). Educating future leaders in patient safety. Journal of Multidisciplinary Healthcare, 7, 381-388. https://doi.org/10.2147/JMDH. S53792.

Nie, J.-B. (2011). Medical ethics in China. Routledge: London and New York.

Turner, L. (2009). Anthropological and sociological critiques of bioethics. Journal of Bioethical Inquiry, 6(1), 83-98. 
World Health Organization. (2009a). Patient safety curriculum guide (specifically for medical schools). Geneva: WHO. http://www.who.int/patientsafety/ education/curriculum_guide_medical_schools/en/.

World Health Organization. (2009b). Conceptual framework for the international classification of patient safety. Geneva: WHO. http://www.who.int/ patientsafety/implementation/taxonomy/ICPS-report/en/.

World Health Organization. (2011). Patient safety curriculum guide (for all health professions). Geneva: WHO. http://www.who.int/patientsafety/ education/mp_curriculum_guide/en/.

Open Access This chapter is licensed under the terms of the Creative Commons Attribution-NonCommercial-NoDerivatives 4.0 International License (http:// creativecommons.org/licenses/by-nc-nd/4.0/), which permits any noncommercial use, sharing, distribution and reproduction in any medium or format, as long as you give appropriate credit to the original author(s) and the source, provide a link to the Creative Commons license and indicate if you modified the licensed material. You do not have permission under this license to share adapted material derived from this chapter or parts of it.

The images or other third party material in this chapter are included in the chapter's Creative Commons license, unless indicated otherwise in a credit line to the material. If material is not included in the chapter's Creative Commons license and your intended use is not permitted by statutory regulation or exceeds the permitted use, you will need to obtain permission directly from the copyright holder.

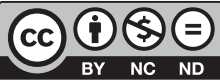

